American Journal of Applied Sciences 7 (3): 420-427, 2010

ISSN 1546-9239

(C) 2010Science Publications

\title{
Orthogonal Frequency Division Multiplexing Timing Synchronization in Multi-Band Orthogonal Frequency Division Multiplexing Ultra-Wideband Systems
}

\author{
${ }^{1}$ Aymen M. Karim, ${ }^{1}$ Md. Shabiul Islam and ${ }^{2}$ Masuri Othman \\ ${ }^{1}$ VLSI LAB, Institute of Microengineering and Nanoelectronics, \\ ${ }^{2}$ Department of Electrical, Electronic and Systems Engineering, Faculty of Engineering, \\ National University of Malaysia, 43600 UKM, Bangi, Selangor, Darul Ehsan, Malaysia
}

\begin{abstract}
Problem statement: MB-OFDM UWB systems have attractive features and received a great attention. It has the capability of sending/receiving data at rates in access of $100 \mathrm{Mbits} \mathrm{sec}^{-1}$. Owing to the sensitivity of OFDM systems to synchronization errors, a fast synchronization algorithm is required to discern for the start of OFDM symbol. Existing approaches to OFDM time synchronization rely on their proposed preamble. Approach: In this study, a comprehensive algorithm for Orthogonal Frequency Division Multiplexing (OFDM) timing synchronization suitable for MultiBand OFDM Ultra-Wideband (MB-OFDM UWB) systems was proposed. Firstly, we proposed a technique for packet detection in the multi-band scenario. Secondly, a new timing metric for timing synchronization is proposed. Then, we propose a technique for frame synchronization. Finally, time frequency code identification and low-complexity timing refinement technique was proposed. Results: The performance of the timing synchronization (coarse synchronization and timing refinement) is evaluated in terms of Mean Squared Error (MSE) in MB-OFDM UWB Channel Models (CM1, CM2, CM3 and CM4). It was found that the proposed method performs better than existing approaches. Conclusion: A suitable timing synchronization steps for MB-OFDM UWB systems was proposed. The proposed method can be applied on any preamble signal.
\end{abstract}

Key word: Multi-band OFDM ultra-wide band systems, time synchronization

\section{INTRODUCTION}

OFDM has been receiving a great interest and has been adopted in many standards such as (Batra et al., 2004) as a base-band modulation technique in the form of MB-OFDM UWB to achieve rates in excess of 100 Mbits $\sec ^{-1}$ at a distance of $10 \mathrm{~m}$. The Multi-Band OFDM system divides the UWB spectrum (3.1-10.6 GHz) into $528 \mathrm{MHz}$ wide sub-bands and uses OFDM modulation to transmit the information in each sub-band.

Due to the sensitivity of OFDM signal against the timing error, a reliable synchronization algorithm is required in order to cope in harsh channel conditions. The synchronization algorithms in literatures are mainly based on sending a training sequence which has a periodic property. At the receiver, autocorrelation is performed to detect the start of the OFDM symbol through maximum value searching.

In this study, we propose a low complexity timing synchronization method suitable to work under harsh channel environment such as MB-OFDM UWB systems channel models (Lai et al., 2007) in an attempt to enhance the MSE.

\section{MATERIALS AND METHODS}

\section{System model:}

IEEE 802.15.3a preamble: The Physical Layer Convergence Protocol (PLCP) preamble in the IEEE 802.15-03 proposal consists of three parts (Batra et al., 2004): Packet Synchronization (PS) sequence, Frame Synchronization (FS) sequence and Channel Estimation (CE) sequence. PS sequence consists of 21 repeated periods denoted as PS0, PS1, ..., PS20 as shown in Fig. 1.

Each piconet uses a distinct time domain sequence (four piconets). After repetition, each period is preappended by 32 zero samples and by appending a guard interval of 5 zero samples resulting in $\mathrm{M}=165$ samples of one PS period. This portion of the preamble can be

Corresponding Author: Aymen M. Karim, VLSI LAB, Institute of Microengineering and Nanoelectronics, National University of Malaysia, 43600 UKM, Bangi, Selangor, Darul Ehsan, Malaysia Tel: 603-89265861 Fax: 603-89216146 
used for packet detection, coarse NFO estimation and coarse symbol timing. The FS sequence part consists of 3 periods denoted as FS0, FS1 and FS2. Each FS period is a PS but with negative sign. This part of the preamble can be used for frame synchronization and Time Frequency Code (TFC) identification. Finally, the CE sequence consists of $6 \mathrm{CE}$ periods denoted as CE0, $\mathrm{CE} 1, \ldots, \mathrm{CE} 5$. This part is used to estimate the channel frequency response, fine frequency offset estimation and fine symbol timing.

Signal and channel model: A block diagram of a complete baseband transmit-receive general OFDM system is illustrated in Fig. 2. The transmitted baseband signal after IFFT operation is given below:

$\mathrm{s}_{\mathrm{m}}=\frac{1}{\sqrt{\mathrm{N}}} \sum_{\mathrm{n} \in \mathrm{I}} \mathrm{D}_{\mathrm{n}} \mathrm{e} \frac{\mathrm{j} 2 \pi \mathrm{nm}}{\mathrm{N}}, 0 \leq \mathrm{m} \leq \mathrm{N}-1$

Where:

$$
\begin{aligned}
& \mathrm{N}=\text { The FFT length } \\
& \mathrm{I}=\mathrm{A} \text { set of subcarriers indices }
\end{aligned}
$$

After cyclic prefix insertion of length $\mathrm{N}_{\mathrm{CP}}$ samples, the transmitted signal is time-frequency interleaved on three frequency bands (denoted as band1, band 2 and band3) according to a predefined kernel or TFC. There is a distinct TFC for each PS sequence.

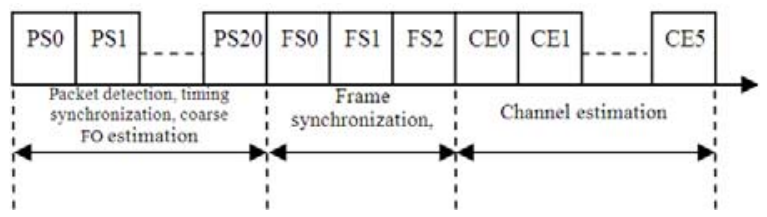

Fig. 1: Preamble structure in (Batra et al., 2004)

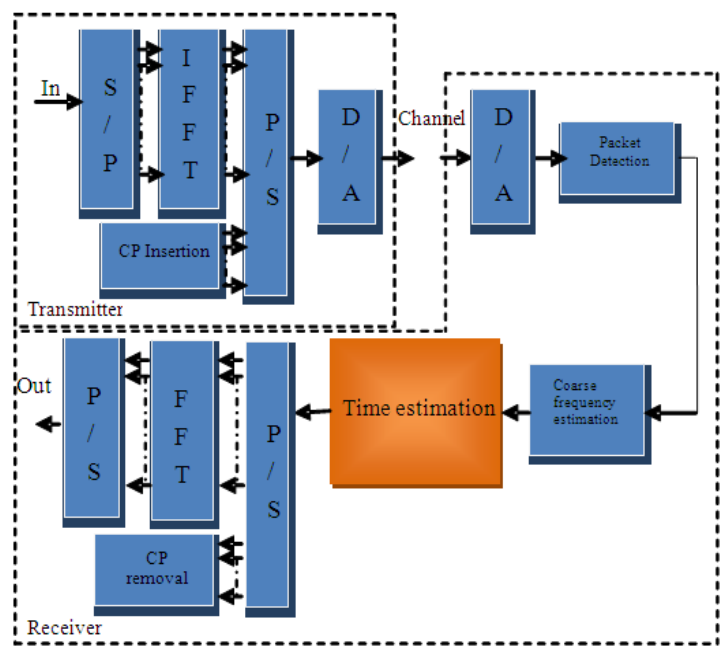

Fig. 2: Block diagram of OFDM systems
The interleaved transmitted signal passes through IEEE 802.15.3a channel model which is a modified version of Saleh and Valenzuela (1987) model. There are four UWB channels models denoted as CM1, CM2, CM3 and CM4, based on the Line-Of-Sight (LOS) existence and the distance between the transmitter and receiver. The channel impulse response is expressed as follows (Lai et al., 2007):

$$
\mathrm{h}(\mathrm{t})=\chi \sum_{\mathrm{l}=0}^{\mathrm{L}} \sum_{\mathrm{k}=0}^{\mathrm{K}} \alpha_{\mathrm{k}, 1} \delta\left(\mathrm{t}-\mathrm{T}_{1}-\mathrm{t}_{\mathrm{k}, 1}\right)
$$

Where:

$a_{k, 1} \quad=$ Multipath gain coefficients

$\mathrm{T}_{1} \quad=$ The delay of the $1^{\text {th }}$ cluster

$\mathrm{T}_{\mathrm{k}, 1}=$ The delay of the $\mathrm{k}^{\text {th }}$ ray in the $\mathrm{l}^{\text {th }}$ cluster

$\delta() \quad=$. The Dirac's delta function

$\chi \quad=$ Log-normal shadowing

$(\mathrm{L}+1)=$ The number of arrival clusters and

$(\mathrm{K}+1)=$ the number of arrival rays within a cluster

More details of the channel model can be found in (Lai et al., 2007).

Figure 3 illustrates one realization of each channel type defined in (Lai et al., 2007), i.e., CM1, CM2, CM3 and CM4 with $528 \mathrm{MHz}$ sampling rate. As it is shown, the first path is not necessary the strongest path with the worst case of CM4 where no LOS is available with a delay spread. The noisy baseband received signal samples in band $\mathrm{q}$ is given as:

$$
\mathrm{r}(\mathrm{d})=\mathrm{e}^{\mathrm{j} 2 \pi \epsilon_{\mathrm{q}} \mathrm{d} / \mathrm{N}} \chi \sum_{\mathrm{l}=0}^{\mathrm{L}} \sum_{\mathrm{k}=0}^{\mathrm{k}} \mathrm{a}_{\mathrm{k}, \mathrm{l}} \mathrm{s}\left(\mathrm{d}-\mathrm{T}_{1}-\mathrm{t}_{\mathrm{k}, \mathrm{l}}\right)+\mathrm{w}(\mathrm{d})
$$

Where:

$\epsilon_{\mathrm{q}}=$ The NFO in band $\mathrm{q}$

$\mathrm{s}(\mathrm{d})=$ The transmitted signal and

$\mathrm{w}(\mathrm{d})=$ The noise sample
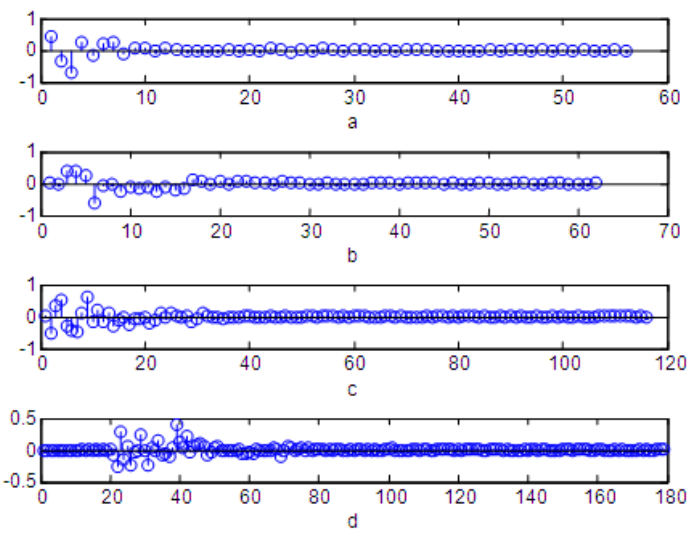

Fig. 3: IEEE 802.15-03 channel model realization. (a) CM1; (b) CM2; (c) CM3 and (d) CM4 
The received signal is then digitized. Afterward, packet detection is achieved to discern for packet existence. Then coarse frequency estimation is carried out to compensate for receiver-transmitter oscillators misalignment. The next block, time estimation, is highlighted using different color to show where present study is focused which has to be done reliably to detect the start of FFT window. Finally, reverse operations are performed to extract the original data $\left(D_{n}\right)$ including post FFT processing such as channel estimation, fine time and frequency synchronization.

Schmidl's synchronization scheme and its improvements: Schmidl and Cox (1997) proposed a synchronization scheme based on a preamble of two identical parts. Normalized correlation over the second part energy is computed and the peak of correlation is considered as the point where the signal starts. The normalized correlation is given by:

$$
\mathrm{M}(\mathrm{d})=\frac{|\mathrm{R}(\mathrm{d})|^{2}}{\mathrm{G}(\mathrm{d})^{2}}
$$

Where:

$$
\begin{aligned}
& R(d)=\sum_{m=0}^{N / 2-1} r *(d+m) r(d+m+N / 2) \\
& G(d)=\sum_{m=0}^{N / 2-1}|r(d+m+N / 2)|^{2}
\end{aligned}
$$

Due to the Cyclic Prefix (CP) appended at the beginning of each symbol, the mean squared error of Schmidl scheme is high as it is shown in (Minn et al., 2000).

Minn et al. (2000) proposed a method to reduce the high MSE of Schmidl by introducing a pilot with more than two symmetrical parts. Moreover, Schmidl method is improved in (Park et al., 2003; Ren et al., 2005). Although the improvements in mean squared error over Schmidl method is very high, they are not applicable only on the specified preamble they propose as it is discussed in (Kang et al., 2008). As a solution, Kang et al. (2008) came with a cross-auto-correlation method based on the given preamble sequence. It shows very good performance even when applied on the previously proposed preamble such as (Park et al., 2003; Ren et al., 2005). However, as it will be shown, Kang's method doesn't perform very well in severe channel environment such as CM4.

Another cross-correlation based approach is proposed by Awoseyila et al. (2009). It exploits
Schmidl preamble and is based computing the cross correlation of the received signal with the known preamble at the receiver. Due to the symmetry of the preamble, the cross-correlation metric has one major peak and two minor peaks. Therefore, autocorrelation of the received signal is computed in order to filter the cross-correlation metric. The result is a metric with only one major peak. Furthermore, the resulting metric is compared with a threshold starting from $\chi$ samples behind the peak of the metric. If the metric is larger than the threshold, the receiver declares that the current sample is the start of the OFDM window. The threshold value computation is derived as follows:

$$
\begin{aligned}
& \text { th }=\sqrt{-\operatorname{In}\left(\frac{\mathrm{P}_{\mathrm{f}}}{\chi}\right)} 2 \hat{\sigma}^{2}, \hat{\sigma}^{2}=\sqrt{\frac{2}{\pi}}\left(\frac{\text { mean }}{\mathrm{d}}\left\{\left|\mathrm{R}_{\mathrm{C}}(\mathrm{d})\right|\right\}\right) \\
& \mathrm{d} \in\left\{\mathrm{d}_{\text {max }}-\frac{\mathrm{N}}{2}+\chi+1, \mathrm{~d}_{\text {max }}-\chi-1\right\}
\end{aligned}
$$

Where:

$$
\begin{aligned}
& \mathrm{R}_{C}(\mathrm{~d})=\sum_{\mathrm{m}=0}^{\mathrm{N}-1} \hat{\mathrm{r}}(\mathrm{d}+\mathrm{m}) \mathrm{P}(\mathrm{m}) \\
& \mathrm{d}_{\max }=\max _{\mathrm{d}}\left(\left|\mathrm{R}_{\mathrm{c}}(\mathrm{d})\right|\right)
\end{aligned}
$$

Where:

$\mathrm{P}_{\mathrm{f}} \quad=$ The desired probability of false alarm

$\left|\mathrm{R}_{\mathrm{C}}(\mathrm{d})\right|=$ The absolute value of the filtered crosscorrelation metric

$\mathrm{d}_{\max }=$ The maximum value of the cross-correlation metric and

$\hat{\mathrm{r}}(\mathrm{d}) \quad=$ The corrected received signal for frequency offset error

False alarm occurs when the metric value is larger than th where $d$ is not the correct FFT window beginning and missed detection occurs when the metric value is less than th at the correct FFT widow beginning.

Despite the significant improvement introduced by the latest scheme, in many cases, the channel condition is unknown. This problem imposes a major difficulty when $\chi$ has to be specified or estimated. As an example, as it is illustrated in Fig. 3, CM1 and CM4 are notably different, where $\chi$ for CM4 need to be larger than that one of CM1. In other words, a universal scheme is required that can be applied and perform well for different channel types. 


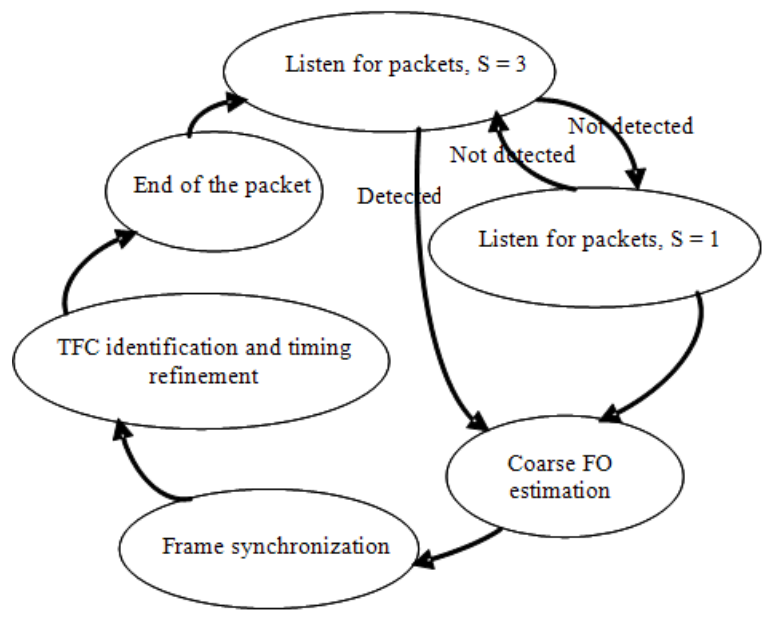

Fig. 4: Synchronizer state diagram

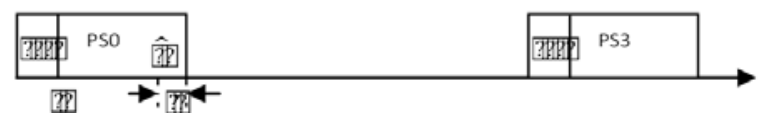

Fig. 5: The received signal in band1 according to the time-frequency code number 1 in (Batra et al., 2004)

Proposed timing synchronizer scheme: The task of timing synchronization is to find the start of the channel estimation sequence. Our synchronizer consists of the states illustrated in Fig. 4, details of each state is given later on.

Packet detection: The PS sequence periodicity is exploited to discern for signal presence. The periodicity of the PS sequence depends on the TFC used. The proposed timing metric for packet detection is given below:

$$
\mathrm{M}_{\mathrm{C}}(\mathrm{d})=\frac{|\mathrm{R}(\mathrm{d})|^{2}}{\mathrm{G}(\mathrm{d})^{2}}
$$

where, $R(d)$ is the as in (5) and G(d) is the same as in (6) with only one difference, the distance between two repeated parts is SM where $\mathrm{S}$ is the number of symbols separating two identical symbols. This metric is utilized in our proposed synchronizer as follows. The receiver is tuned on band number 1 and it listens for packets in this band for a certain time by calculating the timing metric in (1) for $S=3$. If the timing metric, i.e., $M(d)$, exceeds a predefined threshold $\mathrm{h}_{1}$, then the receiver announces the presence of a packet. The TFC number for this case is either 1 or 2 . If $M(d)$ does not exceeds th ${ }_{1}$, then $M(d)$ is calculated for $\mathrm{S}=1$ for a certain time. If its value exceeds $\mathrm{h}_{1}$, the receiver announces the presence of a packet and sets $S=6$ and listens for packets in band number for reasons will be shown later. TFC in this case is either number 3 or 4 . If $M(d)$ does not exceed $\mathrm{th}_{1}$, it sets $\mathrm{S}=1$ and continue until a packet is detected.

The complexity of (4), (5) and (6) can be reduced by a factor of $\omega$ as follows:

$$
\begin{aligned}
& R(d)=\sum_{m=0}^{N / \omega-1} r *(\omega \times d+\omega \times m) r(\omega \times d+\omega \times m+s \times M) \\
& G(d)=\sum_{m=0}^{N / \omega-1}|r(\omega \times d+\omega \times m+s \times M)|^{2}
\end{aligned}
$$

Unlike the synchronizer in (Liu and Lee, 2006), this synchronizer can be used for the sequence illustrated in Fig. 1, where the synchronizer in (Liu and Lee, 2006) averages fewer samples when the error is as shown in Fig. 5. Furthermore, the synchronizer in (Liu and Lee, 2006) does not consider that the received signal is interleaved in three bands.

In Fig. 5, $\theta$ is the correct timing, $\hat{\theta}$ is the timing error, $\mathrm{t}=\mathrm{N}-(\hat{\theta}-\theta)$ and $\mathrm{N}=128$ in (Batra et al., 2004). ZP represents the zero padded cyclic prefix. For the synchronizer in (Liu and Lee, 2006), only t samples are considered in the correlation as the synchronizer jumps each samples and $(\hat{\theta}-\theta)$ samples are considered for the previous correlation value which renders the synchronizer unreliable in low SNR when $t$ is very small and the synchronizer is reduced by a factor of $\omega$.

Moreover, our synchronizer can be evaluated recursively as follows:

$$
\begin{aligned}
R(d+1)= & R(d)+r_{\omega \times(d+1)}^{*}+\omega \times\left(\operatorname{fix}\left(\frac{N}{\omega}\right)-1\right) \\
& r_{\omega \times(d+1)}+\omega \times\left(\operatorname{fix}\left(\frac{N}{\omega}\right)^{-1}\right)+S \times M \\
& -r_{\omega \times d}^{*} r_{\omega \times d+S \times M}
\end{aligned}
$$

where, fix $(x)$ represents rounding towards minus infinity. The same goes for $G(d)$.

From Fig. 1, $\omega$ should be a number divisible by $\mathrm{S} \times \mathrm{M}$, i.e., $\mathrm{S} \times 165$.

NFO estimation: NFO estimation and correction used in our proposal is to correct for the error induced by NFO before our synchronizer switches to TFC identification state. The relationship between the NFO in band number $\mathrm{q}$ is given by:

$$
\epsilon_{\mathrm{q}}=\mathrm{b}_{\mathrm{q}} \in
$$

$\left[b_{1} b_{2} b_{3}\right]=\left[\frac{13}{16}, \frac{15}{16}, \frac{17}{16}\right]$ 
The maximum value of NFO is determined as follows. The total frequency deviation during down conversion is given as $4222 \pm 20=84.48 \mathrm{kHz}$. The whole transmit-receive process introduces an overall carrier frequency error of $\pm 168.96 \mathrm{kHz}$. Normalizing this value with respect to the sub-carrier spacing, $\Delta \mathrm{f}=4.125 \mathrm{MHz}$ results in $\in= \pm 0.04096$. Therefore, the maximum NFO is $\in_{\mathfrak{s}}= \pm 0.0435$. The NFO estimate is obtained from:

$\hat{\epsilon}_{\mathrm{q}} \frac{\mathrm{N}}{2 \pi \times 3 \mathrm{M}} \operatorname{angle}(\mathrm{R}(\mathrm{d}))$

After that the signal is corrected by multiplying each sample by $\mathrm{e}^{-\mathrm{j} 2 \pi \hat{\epsilon}_{\mathrm{q}} \mathrm{d} / \mathrm{N}}$ as follows:

$\hat{r}(d)=r(d) e^{-\frac{j 2 \pi \hat{\epsilon}_{q} d}{N}}$

where, $d$ is the sample number.

Frame synchronization: Up to this point, the start of an OFDM symbol is coarsely known at the receiver. But the whole frame timing is still vague (i.e., the start of FS which is df ). As it is discussed previously, FS is the as PS but with negative sign. This can be exploited to discern the CE start by observing $\mathrm{R}(\mathrm{d})$. As it is shown in Fig. 6, R(d) for TFC number 1 and 2, goes below zero when $d$ is at PS20. In our design, receiver tracks $\mathrm{R}(\mathrm{d})$, if it is less than a predefined threshold $\mathrm{th}_{2}$, the minimum of the next $\mathrm{R}(\mathrm{d})$ samples ( $\mathrm{N}$ samples) is found and added to $\mathrm{S} \times \mathrm{M}$ i.e.:

$\widehat{\mathrm{d} f}=\min _{\mathrm{d}} \mathrm{R}(\mathrm{d})+\mathrm{S} \times \mathrm{M}$

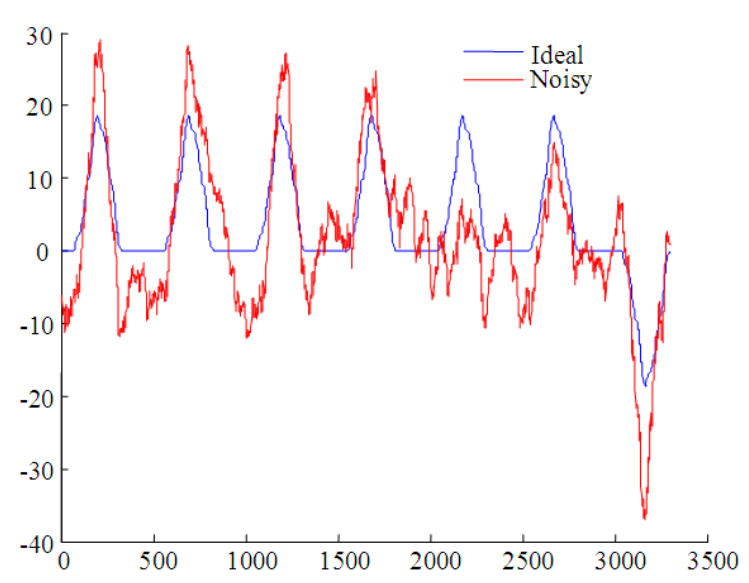

Fig. 6: R(d) for TFC number 1 and 2
If $\mathrm{R}(\mathrm{d})$ does not plunge under, a false alarm occurs and the receiver goes to listen for a packet state (Fig. 4). The threshold value $\left(\mathrm{th}_{2}\right)$ is determined depending on the value of $\mathrm{R}(\mathrm{d})$ during packet detection through simulation.

Figure 7 illustrates R(d) for TFC number 3 and 4. As we can see, the same previous steps can be applied in this case to find the start of FS.

At this synchronization state, received signal must be corrected for NFO. Otherwise, the repeated symbols (periodicity) of the received signal will be in negative sign when NFO is near 0.05. In this case the receiver has to track for sign changes in $\mathrm{R}(\mathrm{d})$ rather than its falling below a certain threshold.

TFC identification and timing refinement: Before proceeding to the next receiver tasks, received signal TFC must be identified. It is known up to now that TFC belongs either to $1,2,3$ or 4 . The next steps are applied for the first case. The same steps can be applied for the second one. After frame synchronization, the $2 \mathrm{~N}$ samples after $d \widehat{\mathrm{f}}-\mathrm{C}$ and $\mathrm{d} \hat{\mathrm{f}}-\mathrm{C}+\mathrm{SM}$ are stored, where $\mathrm{C}$ is chosen to set back the timing on the packet synchronization sequence.

Cross correlation between the NFO corrected received signal and the FS sequence for TFC no. 1 and 2 is performed as follows:

$\mathrm{R}_{\mathrm{C}}(\mathrm{d})=\left|\sum_{\mathrm{m}=0}^{\mathrm{N}-1} \hat{\mathrm{r}}(\mathrm{d}+\mathrm{m}) \mathrm{P}(\mathrm{m})\right|^{2}$

where, $\mathrm{P}(\mathrm{m})$ is the samples of PS sequence as defined in (Foerster et al., 2001). Two sequences of $\mathrm{R}_{\mathrm{C}}(\mathrm{d})$ are obtained, one for TFC no. 1 and TFC no. 2.

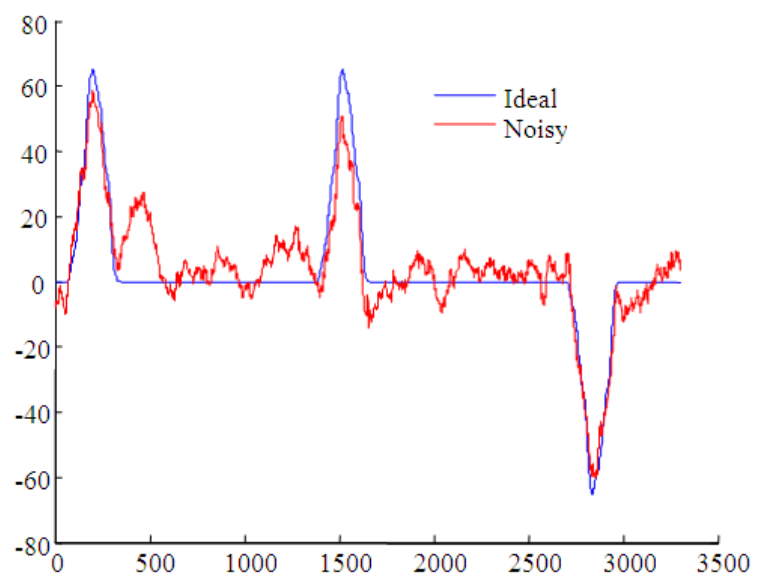

Fig. 7: R(d) for TFC number 3 and 4 
There maximum values are obtained by feeding the output of the creator into a comparator to determine the maximum value. These maximum values are compared with each other, if the one corresponds to TFC no. 1 is larger than the other, then TFC will be identified as TFC no. 1, the opposite applies when it is less.

The maximum value of cross correlation does not carry information about the start of FFT window when the first arrival path is not necessary the strongest path. Therefore, a robust synchronization method is required to discern for the start of the FFT window. As we mentioned earlier, the proposed methods in the literature are workable only on a specially designed preamble. In present study, we devise the temporal correlation of the cross correlation value in (22) as follows:

$$
\begin{gathered}
\mathrm{P}_{\mathrm{x}}(\mathrm{d})=\sum_{\mathrm{m}=0}^{\mathrm{L}-1}\left|\mathrm{R}_{\mathrm{C}}(\mathrm{d}+\mathrm{m})\right|^{2}+\left|\mathrm{R}_{\mathrm{C}}(\mathrm{d}+\mathrm{m}+\mathrm{S})\right|^{2} \\
\mathrm{~d} \in\left\{\mathrm{d}_{\text {max }}-\mathrm{N} / 2, \mathrm{~d}_{\text {max }}+\mathrm{N} / 2\right\}
\end{gathered}
$$

and the time estimate is given as:

$$
\hat{d}=\max _{d} P_{x}(d)
$$

$\mathrm{L}$ is chosen according to the channel path number. The correlation is not performed over the whole crosscorrelation value, but only on the two sets of the $2 \mathrm{~N}$ samples to reduce complexity. In order to reduce the complexity furthermore, cross correlation complexity can be reduced by taking only the sign bit of the known sequence FS and multiply it by the stored sequence, i.e., $\hat{\mathrm{r}}(\mathrm{d})$. This will relax our cross correlation by replacing the 128 complex multiplier with a sign flipper. Moreover, the complexity can be reduced by taking only the real part of the $2 \mathrm{~N}$ samples.

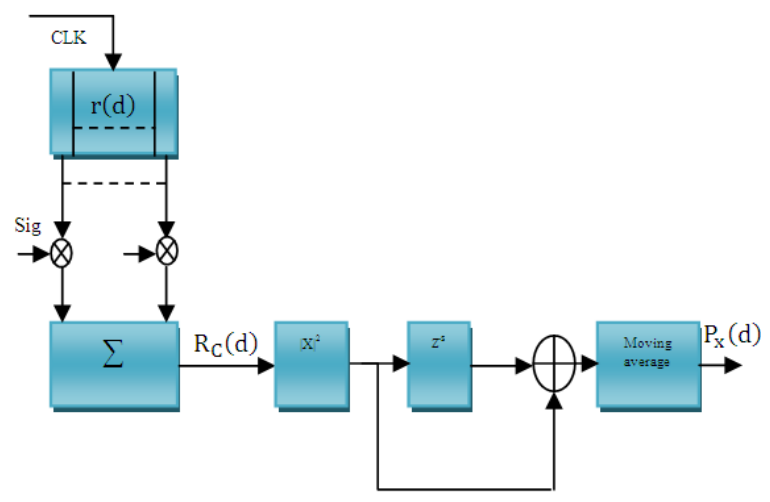

Fig. 8: Proposed method block diagram
Figure 8 shows a block diagram of our cross correlate and comparator. A clocked shift-right register is used, each sample is passed or sign flipped according to FS. Then, these samples are summed up to produce $\mathrm{R}_{\mathrm{C}}(\mathrm{d})$. The comparator consists of a comparator and gate and shift register.

\section{RESULTS}

At first, performance analysis is investigated for the IEEE 802.11a preamble (IEEE-802.11a-1999, 1999) as in (Kang et al., 2008). Raleigh channel mode with 16 paths is applied with a normalized frequency offset of 0.2 and exponential power profile with average tap power $\rho_{\mathrm{m}}=\exp \left(-\frac{\tau_{\mathrm{m}}}{30}\right) / \sum_{\mathrm{m}=0}^{\mathrm{M}-1} \exp \left(-\frac{\tau_{\mathrm{m}}}{30}\right)$, where $\mathrm{M}=16$. Finally, $P_{\mathrm{f}}=10^{-6}$. We will call this the first case in the upcoming discussion. Afterward, it is investigated for the channel model CM1, CM2, CM3 and CM4 with normalized frequency offset $=0.05$. The parameters such as $\mathrm{CP}$ length and FFT length all are the same as in (Batra et al., 2004) including the PLCP preamble. $P_{f}=10^{-6}$ in Adegbenga method. We will call this case the second case. For both cases, MSE is computed for Kang, Adegbenga, proposed and proposed low complexity (just in the first case using sign flippers instead of multipliers).

Figure 9 illustrates the first case MSE versus SNR in $\mathrm{dB}$ with $\chi=16$ in Adegbenga scheme and $\mathrm{L}=16$ in the proposed method to make fair comparison (i.e., the fails in this channel with high MSE. Due to the false alarm or missed detection resulted by Adegbenga scheme, his MSE is higher than even the low-complexity proposed method.

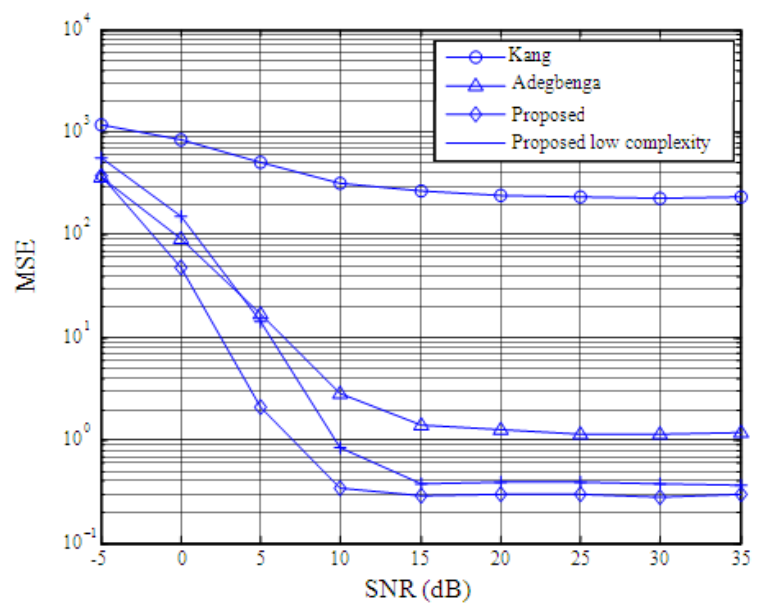

Fig. 9: MSE versus SNR of Kang, Adegbenga, proposed and proposed low complexity scheme in Rayleigh channel 


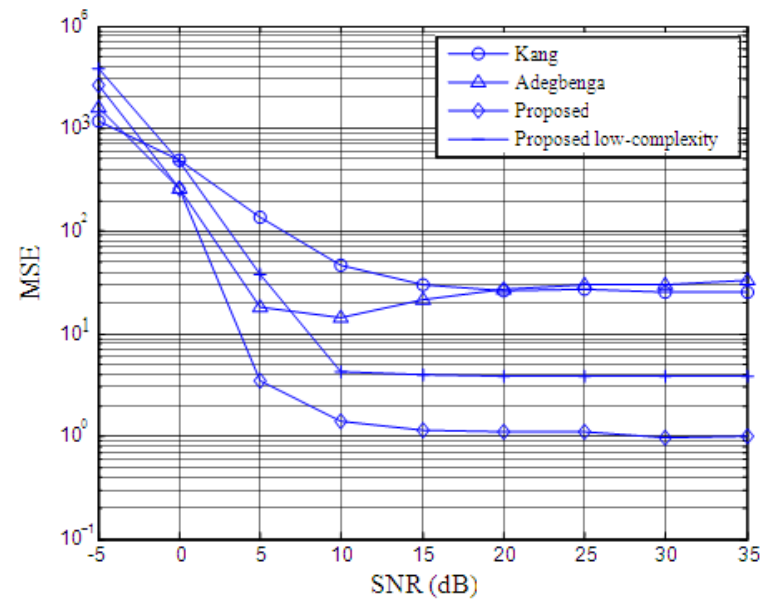

(a)

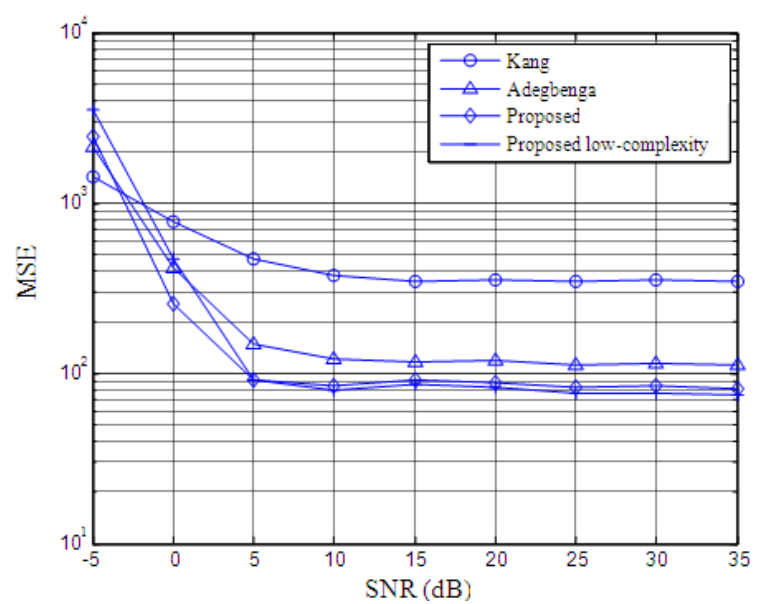

(c)

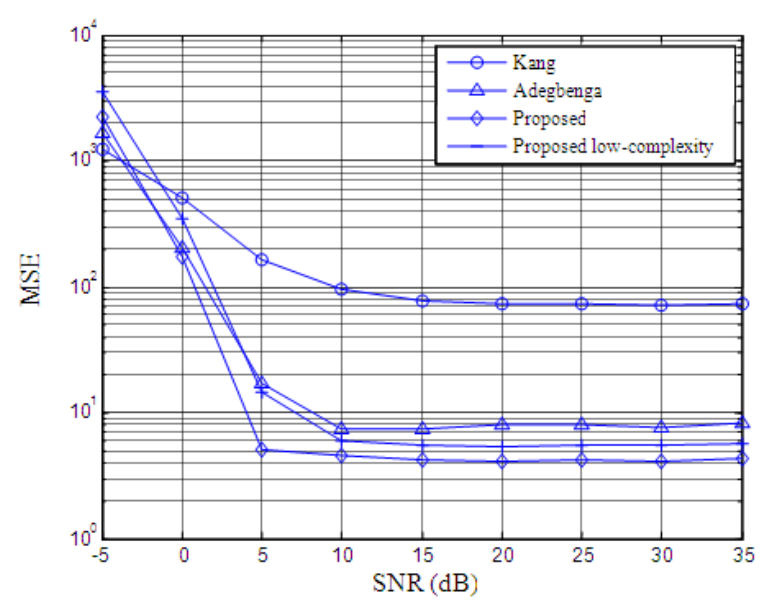

(b)

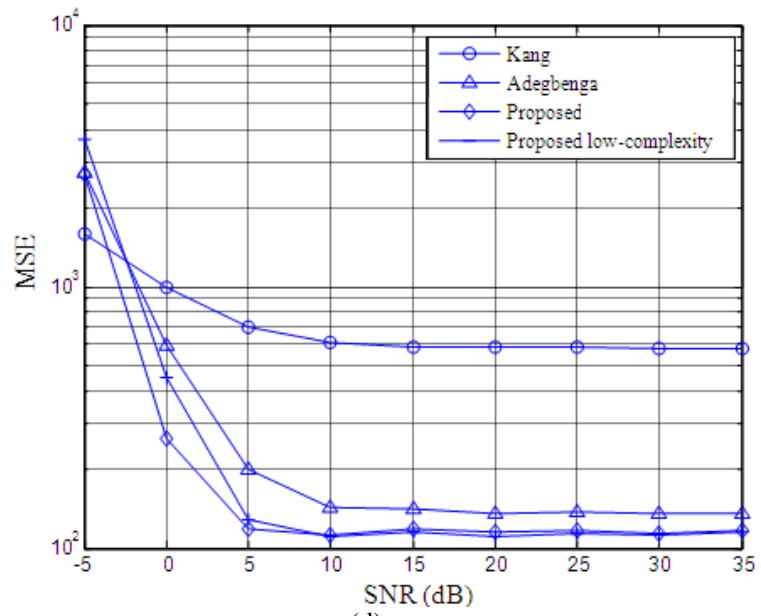

(d)

Fig. 10: MSE versus SNR of Kang's, Adegbenga's, Proposed and Proposed low-complexity method in (a) CM1; (b) $\mathrm{CM} 2$; (c) CM3 and (d) CM4

As for the second case, we set $\chi=\mathrm{L}=10$ in Adegbenga and our scheme in CM1 and CM2 and $\chi=\mathrm{L}$ $=32$ in $\mathrm{CM} 3$ and CM4.

Figure 10 a-d illustrates the computed MSE's versus $\mathrm{SNR}$ in $\mathrm{dB}$ in $\mathrm{CM} 1, \mathrm{CM} 2, \mathrm{CM} 3$ and $\mathrm{CM} 4$ respectively.

\section{DISCUSSION}

As we can see from Fig. 9, Kang's method MSE is very high compared with the proposed method. It is also shown that even for the proposed low-complexity method; our synchronization algorithm performs better than Adegbenga's method. As for the second case, i. e. Fig. 10, Kang's method MSE is very high in all the channel models (CM1, CM2, CM3, and $\mathrm{CM} 4$ ).
Although Adegbenga improves the MSE, it is apparent that our MSE is always lower than that of Adebenga's method even for the low complexity synchronization method.

\section{CONCLUSION}

A reliable synchronization method is proposed suitable for MB-OFDM UWB systems. As it is explained, previously existing methods rely on their proposed preamble. Although this problem is solved in (Kang et al., 2008), the solution has a high MSE in a severe channel environment such as CM4. As it is shown earlier, the method in (Awoseyila et al., 2009) performs very well, but it lacks a mechanism to 
compute $\mathrm{x}$ which is a problem because $\mathrm{x}$ should be low for the channel with LOS such as CM1 and high for non line of site channels and the channel is unknown for the receiver (whether it is $\mathrm{CM} 1, \mathrm{CM} 2$ ).

\section{REFERENCES}

Awoseyila, A.B., C. Kasparis and B.G. Evans, 2009. Robust time-domain timing and frequency synchronization for OFDM systems. IEEE Trans. Consum. Elect., 55: 391-399. DOI: 10.1109/TCE.2009.5174399

Batra, A., J. Balakrishnan, G.R. Aiello, J.R. Foerster and A. Dabak, 2004. Design of a multiband OFDM system for realistic UWB channel environments. IEEE Trans. Microwave Theory and Techniques, 9: 2123-2138. DOI: 10.1109/TMTT.2004.834184

Foerster, J., E. Green, S. Somayazulu and D. Leeper, 2001. Ultra-wideband technology for short-or medium-range wireless communications. Intel Technol. J., Q2: 1-11. http://www.intel.com/technology/itj/q22001/pdf/art 4.pdf

IEEE-802.11a-1999, 1999. Part 11: Wireless LAN Medium Access Control (MAC) and Physical Layer (PHY) Specifications: High-Speed Physical Layer in the $5 \mathrm{GHz}$ Band. IEEE. http://standards.ieee.org/getieee802/download/802. 11-2007.pdf

Kang, Y., S. Kim, D. Ahn and H. Lee, 2008. Timing estimation for OFDM systems by using a correlation sequence of preamble. IEEE Trans. Cons. Elect., 54: 1600-1608. DOI: 10.1109/TCE.2008.4711208
Lai, H.Q., W.P. Siriwongpairat and K.J.R. Liu, 2007. Performance analysis of multiband OFDM UWB systems with imperfect synchronization and intersymbol interference. IEEE J. Select. Top. Signal Process., 1: 521-534. DOI: 10.1109/JSTSP.2007.906650

Liu, H.Y. and C.Y. Lee, 2006. A low-complexity synchronizer for OFDM-based UWB system. IEEE Tans. Circ. Syst. II, Express Briefs, 53: 1269-1273. DOI: 10.1109/TCSII.2006.882804

Minn, H., M. Zeng and V.K. Bhargava, 2000. On timing offset estimation for OFDM systems. IEEE Commun. Lett., 4: 242-244. DOI: 10.1109/4234.852929

Park, B., H. Cheon, C. Kang and D. Hong, 2003. A novel timing estimation method for OFDM systems. IEEE Commun. Lett., 7: 239-241. DOI: 10.1109/LCOMM.2003.812181

Ren, G., Y. Chang., H. Zhang and H. Zhang, 2005. Synchronization method based on a new constant envelop preamble for OFDM systems. IEEE Trans. Broadcast., 51: 139-143. DOI: 10.1109/TBC.2004.842520

Saleh, A. and R. Valenzuela, 1987. A statistical model for indoor multipath propagation. IEEE J. Select. Areas Commun., 5: 128-137. DOI: 07338716/87/0200-0128

Schmidl, T.M. and D.C. Cox, 1997. Robust frequency and timing synchronization for OFDM. IEEE Trans. Commun., 45: 1613-1621. DOI: $10.1109 / 26.650240$ 